\title{
Association Between Treatment Facility Volume, Therapy Types, and Overall Survival in Patients With Stage IIIA Non-Small Cell Lung Cancer
}

Anuhya Kommalapati, MBBS ${ }^{a, b, *}$; Sri Harsha Tella, MBBS a,b,*; Adams Kusi Appiah, PhD; Lynette Smith, $\mathrm{PhD}^{\mathrm{c}}$; and Apar Kishor Ganti, $\mathrm{MD}^{\mathrm{d}}$

\begin{abstract}
Background: There is significant heterogeneity in the treatment of stage IIIA non-small cell lung cancer (NSCLC). This study evaluated the therapeutic and survival disparities in patients with stage IIIA NSCLC based on the facility volume using the National Cancer Database. Methods: Patients with stage IIIA NSCLC diagnosed from 2004 through 2015 were included. Facilities were classified by tertiles based on mean patients treated per year, with low-volume facilities treating $\leq 8$ patients, intermediate-volume treating 9 to 14 patients, and high-volume treating $\geq 15$ patients. Cox multivariate analysis was used to determine the volume-outcome relationship. Results: Analysis included 83,673 patients treated at 1,319 facilities. Compared with patients treated at low-volume facilities, those treated at high-volume centers were more likely to be treated with surgical ( $25 \%$ vs $18 \%)$ and trimodality $(12 \%$ vs $9 \%)$ therapies. In multivariate analysis, facility volume was independently associated with all-cause mortality $(P<.0001)$. Median overall survival by facility volume was 15,16 , and 19 months for low-, intermediate-, and high-volume facilities, respectively $(P<.001)$. Compared with patients treated at high-volume facilities, those treated at intermediateand low-volume facilities had a significantly higher risk of death (hazard ratio, 1.09 [95\% Cl, 1.07-1.11] and 1.11 [95\% Cl, 1.09-1.13], respectively). Conclusions: Patients treated for stage IIIA NSCLC at high-volume facilities were more likely to receive surgical and trimodality therapies and had a significant improvement in survival. J Natl Compr Canc Netw 2019;17(3):229-236 doi: $10.6004 /$ jnccn.2018.7086
\end{abstract}

a Department of Internal Medicine, University of South Carolina School of Medicine, and ${ }^{b}$ Palmetto Health USC Medical Group, Columbia, South

Carolina; and 'Department of Biostatistics, College of Public Health, University of Nebraska Medical Center, and 'Department of Hematology and Oncology, VA Nebraska Western lowa Health Care System and University of Nebraska Medical Center, Omaha, Nebraska.

\section{Background}

Lung cancer is the leading cause of cancer-related death in the United States, attributed to approximately 44.7 deaths per 100,000 persons annually. ${ }^{1}$ An estimated 234,000 cases were newly diagnosed in 2018 in the United States, with 154,000 deaths from the disease. ${ }^{2}$ Non-small cell lung cancer (NSCLC) constitutes approximately $85 \%$ to $90 \%$ of all lung cancers. Among patients with NSCLC, it is estimated that approximately $20 \%$ have locoregional spread and approximately $27 \%$ have stage IIIA disease at initial diagnosis. Stage IIIA disease or locally advanced NSCLC includes a wide variety of subsets, such as T1-T3N2M0, T3N1M0, and T4N0 or N1M0 disease, depending on the size of the primary tumor and location of nodal metastases.

Given the variety of subsets, there has been significant heterogeneity in the treatment of stage IIIA NSCLC, and the exact indications for and role of surgery have been controversial. ${ }^{3-5}$ Most clinical trials of patients with locally advanced disease have included the entire spectrum of patients with stage IIIA NSCLC, and the numbers in individual subsets of categories have been too small to make any definite conclusions on the utility of that particular therapy option in that specific subset of patients.

The Intergroup 0139 study demonstrated an improvement in 5-year progression-free survival and local control with surgical resection after neoadjuvant chemoradiotherapy. ${ }^{6}$ A trend toward improved overall survival (OS) was seen in the surgery group, although this did not reach statistical significance $(27 \%$ vs $20 \%$; odds ratio, $0.63 ; 95 \% \mathrm{CI}, 0.36-1.10$ ). The resectability of T4 or N2 disease is highly dependent on the local expertise of the surgeon and the management of complications by the comprehensive care team. ${ }^{7}$ Moreover,

See JNCCN.org for supplemental online content. 
despite an increased association between toxicity and combined chemoradiotherapy (CRT) in stage IIIA disease, previous studies have shown an advantage for combination CRT. ${ }^{8}$ This survival advantage may be attributed to better management of the complications or toxicities.

Over time, multiple studies have described higher rates of mortality at centers where fewer patients with cancer were treated annually. ${ }^{9}$ We hypothesized that this may also be the case with stage IIIA NSCLC because of the absence of specific uniform treatment patterns. Previous studies have shown interinstitutional variations in therapeutic algorithms for stage IIIA NSCLC. ${ }^{10}$ Therefore, we sought to analyze the association between facility volume, treatment modalities offered, and different risk-adjusted outcomes in patients with stage IIIA NSCLC.

\section{Methods}

\section{Data Source}

Data for patients with stage IIIA NSCLC diagnosed from 2004 through 2015 were obtained from the National Cancer Database (NCDB), which includes $>70 \%$ of all newly diagnosed cancer cases in the United States and Puerto Rico. Because both the facility and patient data are deidentified, this study was deemed exempt from review by the Palmetto Health-University of South Carolina Institutional Review Board.

\section{Study Population}

A total of 1,393,073 patients with NSCLC were identified in the NCDB from 2004 through 2015. Patients with stages of NSCLC other than IIIA $(n=1,249,863)$, multiple malignancies $(n=32,623)$, and unknown length of follow-up $(n=9)$ were excluded. Furthermore, because the NCDB provides data based on the facility at which the malignancy was initially diagnosed and first-line treatment was offered, patients diagnosed at one facility and treated at another were also excluded (class of case " 00 "; $n=15,225$ ). Patients diagnosed in 2015 $(\mathrm{n}=9,700)$ were also excluded from the final OS analysis to allow at least 1 year of follow-up. We selected the "reference date cutoff" as 1 to account for completeness of data $(n=1,980)$. The case selection process is detailed in Figure 1.

\section{Facility Case Volume, Therapeutics, and}

Survival Analysis

After the study cohort was identified, patients with stage IIIA NSCLC were divided into tertiles (defined a priori) based on average annual patient volumes at managing facilities (ie, low-volume facility, lower one-third tertile; intermediate-volume facility, middle one-third; and

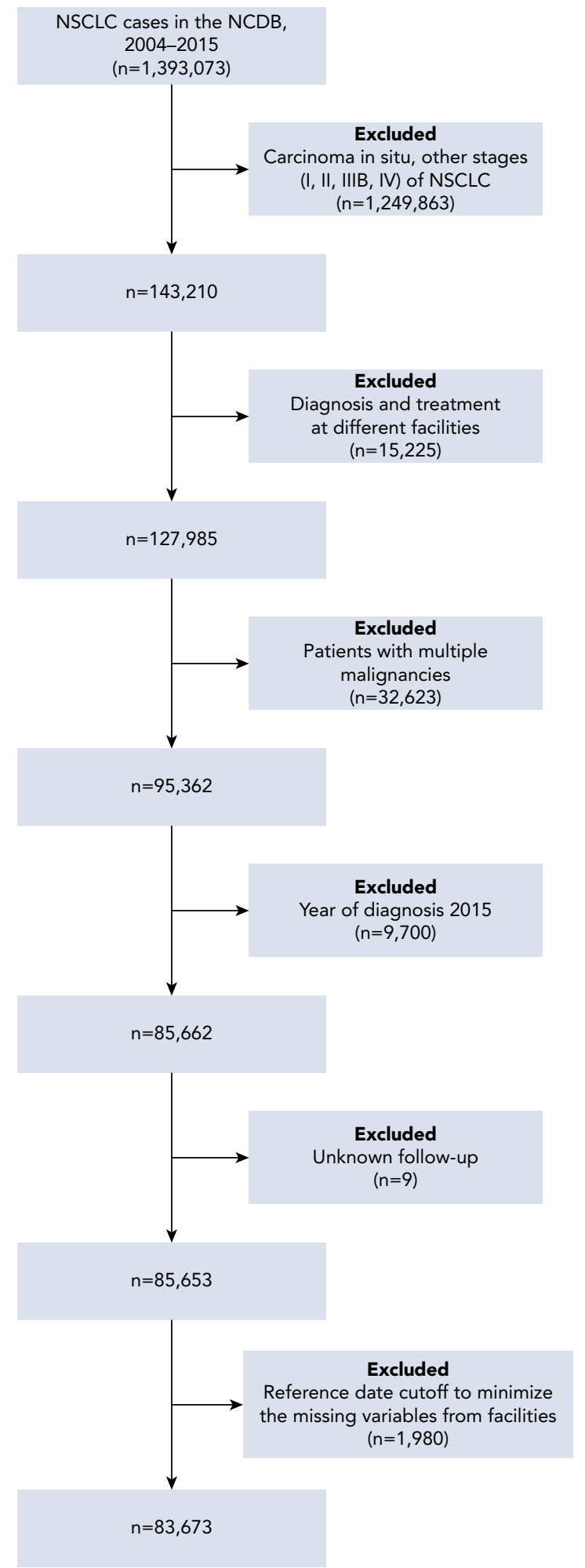

Figure 1. Case selection process for patients with stage IIIA nonsmall cell lung cancer (NSCLC) from the National Cancer Database (NCDB). 
Table 1. Patient and Tumor Characteristics

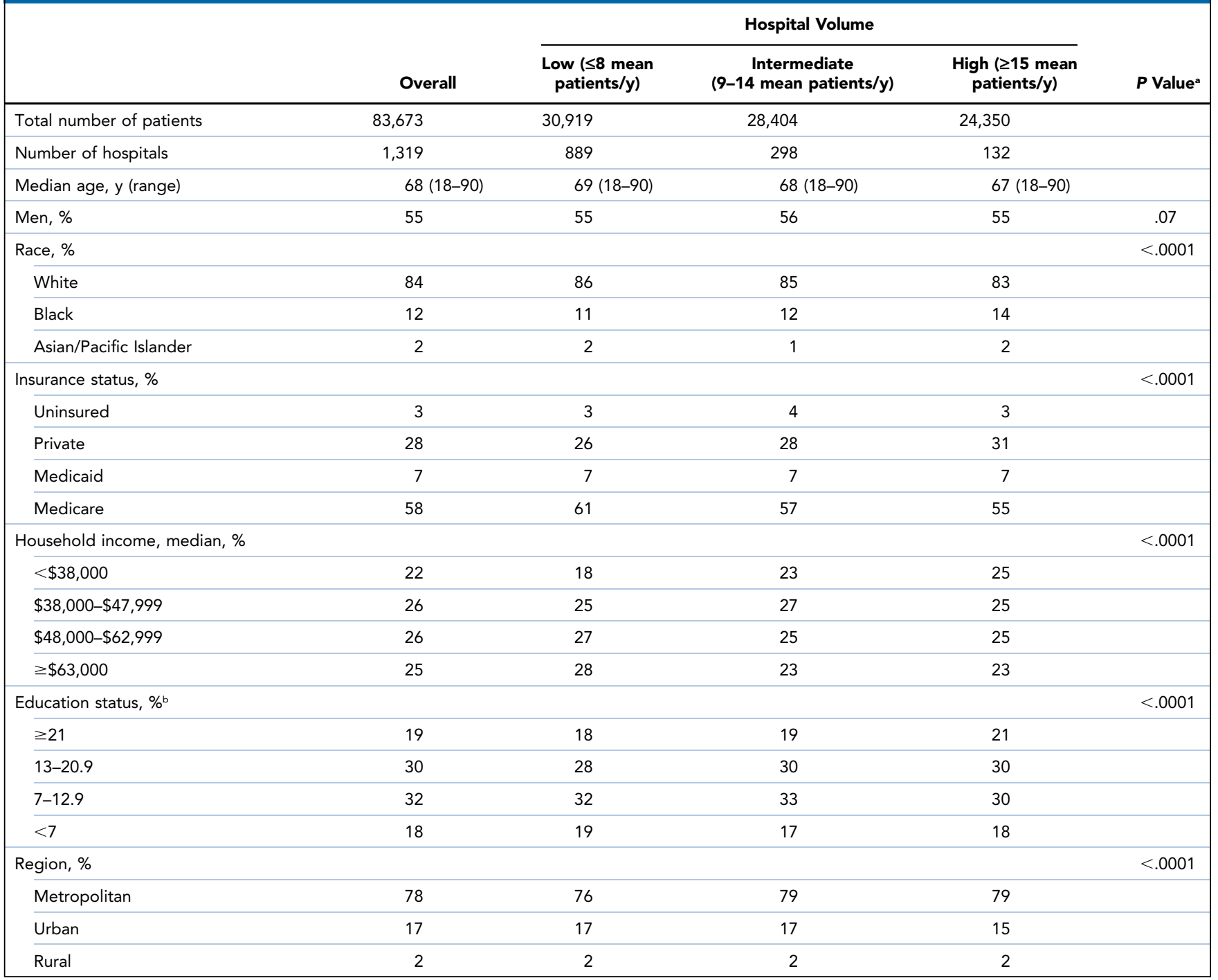

a $P$ value represents statistical differences in baseline characteristics among the cohorts.

(continued on next page)

bPercentage who did not graduate from high school.

high-volume facility, upper one-third) (Table 1). Our primary outcome of interest was OS, defined as the interval between stage IIIA NSCLC diagnosis and death. Patients who were alive at last follow-up were censored from the analysis. Information on type of therapies received and OS based on hospital volume were analyzed from the database.

\section{Covariates Included}

The primary predictor of interest in the analysis was facility volume. Factors that significantly predicted survival in the univariate model were patient demographics (age, sex, race), insurance status (no insurance, private, Medicare, or Medicaid), Charlson-Deyo comorbidity score, tumor characteristics (grade), and type of therapy received (eg, surgery, chemotherapy, and/or radiotherapy) $(P<.01)$. The univariate association of each covariate with OS was assessed using a Cox proportional hazards model (Table 2). Covariates that were significantly associated with OS $(P<.05)$ were included in the multivariable model, and forward stepwise selection was used with a $P$ value cutoff of .10 to exclude the covariates.

\section{Statistical Analysis}

SPSS Statistics, version 24 (IBM Corporation) was used to account for clustering of patients within facilities (based on volume: lower one-third, middle one-third, and higher one-third of patients), and a Cox multivariable 
Table 1. Patient and Tumor Characteristics (cont.)

\begin{tabular}{|c|c|c|c|c|c|}
\hline \multirow{3}{*}{ Charlson-Deyo comorbidity score, \% } & \multirow[b]{2}{*}{ Overall } & \multicolumn{3}{|c|}{ Hospital Volume } & \multirow[b]{2}{*}{$P$ Value } \\
\hline & & $\begin{array}{l}\text { Low ( } \leq 8 \text { mean } \\
\text { patients/y) }\end{array}$ & $\begin{array}{c}\text { Intermediate } \\
\text { (9-14 mean patients/y) }\end{array}$ & $\begin{array}{l}\text { High ( } \geq 15 \text { mean } \\
\text { patients/y) }\end{array}$ & \\
\hline & & & & & $<.0001$ \\
\hline $0-1$ & 87 & 87 & 87 & 88 & \\
\hline $2-3$ & 13 & 13 & 13 & 12 & \\
\hline Tumor grade, $\%$ & & & & & $<.0001$ \\
\hline $1-2$ & 21 & 22 & 22 & 20 & \\
\hline $3-4$ & 36 & 37 & 35 & 35 & \\
\hline Unknown & 43 & 41 & 43 & 45 & \\
\hline T stage, $\%$ & & & & & $<.0001$ \\
\hline T1 & 17 & 16 & 17 & 18 & \\
\hline T2 & 39 & 39 & 39 & 40 & \\
\hline T3 & 28 & 29 & 28 & 27 & \\
\hline T4 & 12 & 12 & 12 & 11 & \\
\hline Nodal status, \% & & & & & $<.0001$ \\
\hline No & 9 & 9 & 9 & 9 & \\
\hline N1 & 10 & 10 & 10 & 10 & \\
\hline N2 & 78 & 78 & 78 & 78 & \\
\hline Therapies received, \% & & & & & $<.0001$ \\
\hline No treatment & 15 & 17 & 15 & 13 & \\
\hline Trimodality & 10 & 9 & 9 & 12 & \\
\hline Surgery only & 5 & 4 & 5 & 6 & \\
\hline Chemotherapy only & 8 & 8 & 8 & 8 & \\
\hline Radiotherapy only & 11 & 11 & 11 & 10 & \\
\hline Chemoradiotherapy & 46 & 46 & 47 & 44 & \\
\hline Other & 5 & 5 & & & \\
\hline RO resection, \% & 17 & 14 & 16 & 21 & $<.0001$ \\
\hline
\end{tabular}

a $P$ value represents statistical differences in baseline characteristics among the cohorts.

bPercentage who did not graduate from high school.

proportional hazards model was used to determine the volume-outcome relationship, adjusting for covariables discussed in the previous section. We expressed nonnormally distributed continuous variables as median and range. One-way analysis of variance was used to compare continuous variables, and chi-square tests were used to compare categorical variables between tertiles. OS was estimated using the Kaplan-Meier method and log-rank tests. Values of $P<.05$ were considered statistically significant.

\section{Results}

\section{Baseline Characteristics and Facility}

Volume Stratification

A total of 83,673 patients with stage IIIA NSCLC diagnosed in 2004 through 2014 and treated at 1,319 facilities that met the inclusion criteria. Median age at diagnosis was 68 years (range, 18-90 years), and 55\% were men. Median annual facility volume was 10 patients per year (range, 1-88 patients per year). Facilities in the cohort were classified into tertiles based on mean patients with stage IIIA NSCLC treated per year: low-volume (tertile 1), $\leq 8$ patients; intermediate-volume (tertile 2), 9 to 14 patients; and high-volume (tertile 3 ), $\geq 15$ patients. Low-, intermediate-, and high-volume facilities accounted for $37 \%(n=30,919), 34 \%(n=28,404)$, and $29 \%(n=24,350)$ of patients, respectively. No clinically meaningful differences in patient demographics and tumor characteristics were noted with respect to hospital volume (Table 1).

Treatment Analysis Based on Facility Volume Significant differences were noted in the management of stage IIIA NSCLC by facility case volume. Patients at 


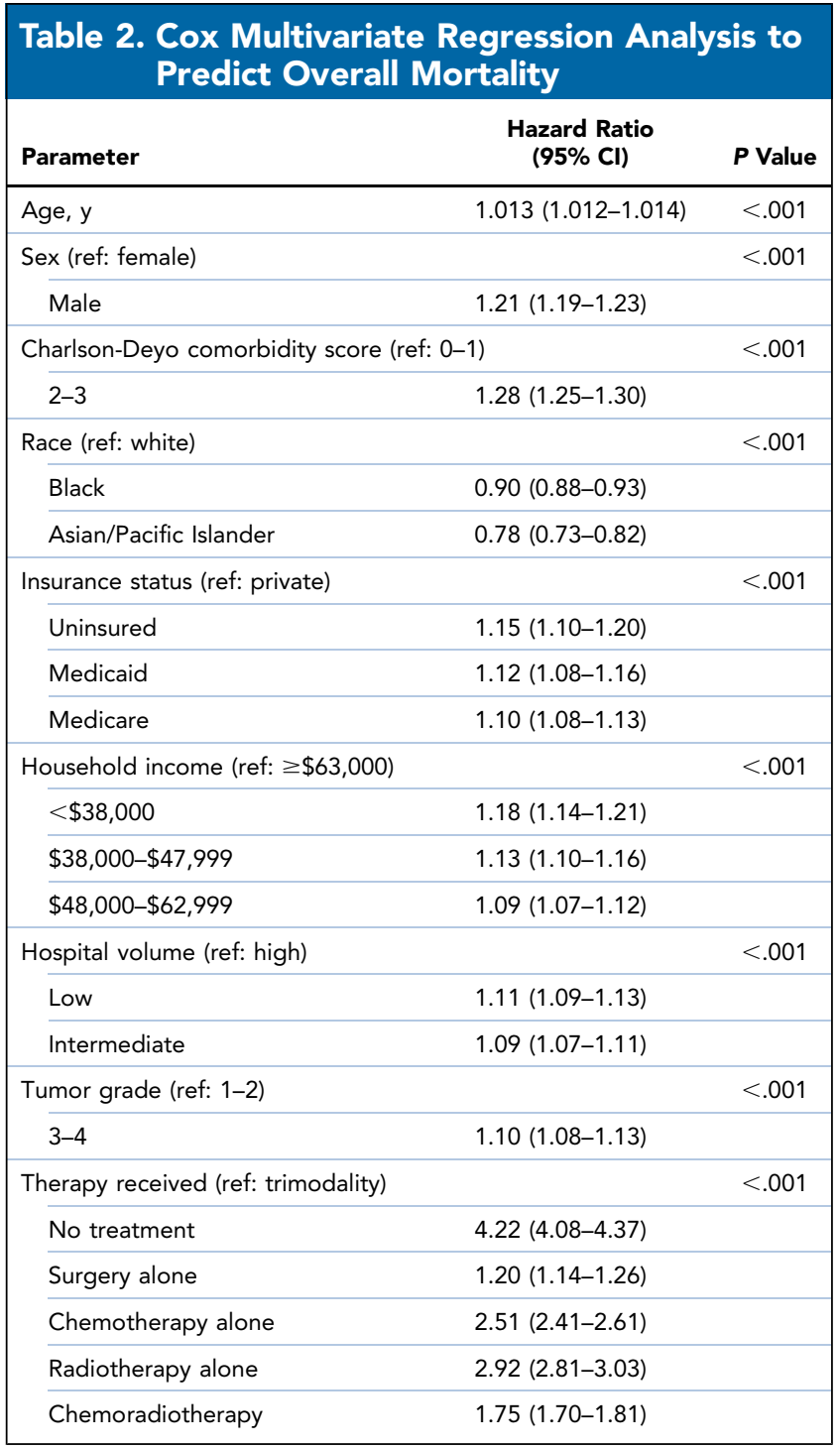

high-volume facilities were more likely to receive some form of therapy ( $87 \%$ vs $83 \%$ and $85 \%$ at low- and intermediate-volume facilities, respectively; $P<.001$ ). Although the percentage of patients who received chemotherapy and radiotherapy was similar among the facilities, patients at high-volume centers were more likely to receive neoadjuvant chemoradiotherapy ( $7 \%$ vs $4 \%$ at low-volume facilities).

Factors that predicted use of surgery in logistic regression analysis were female sex, lower Charlson-Deyo comorbidity score, private insurance, higher education status, lower $\mathrm{T}$ and $\mathrm{N}$ stages, and treatment at highvolume centers (Table 3 ). Approximately $25 \%$ of the patients treated at high-volume facilities received surgical therapy (either alone or in combination) as part of their management plan compared with $20 \%$ and $18 \%$ of

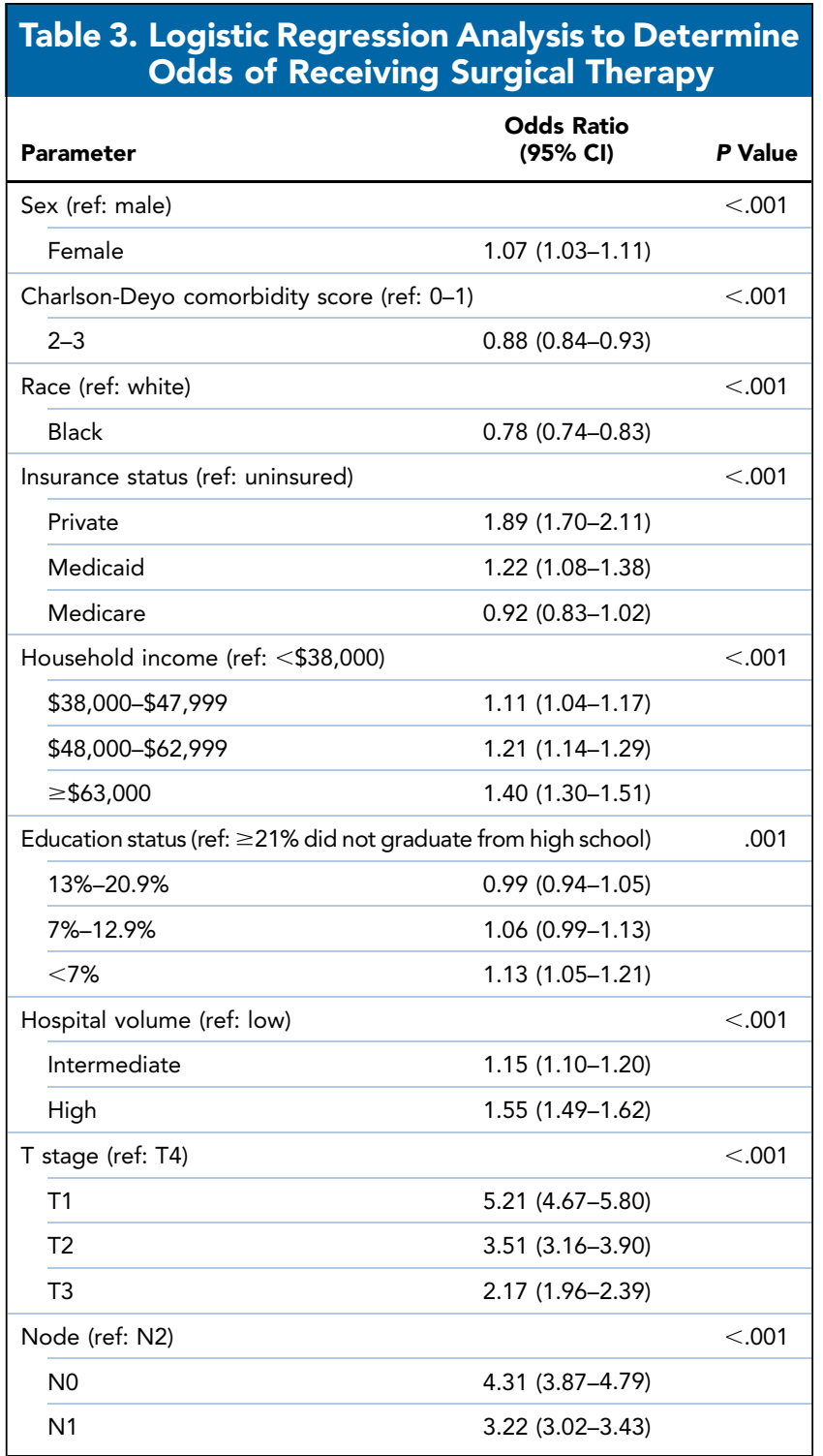

patients treated at intermediate- and low-volume centers, respectively. Patients managed with surgical therapy at high-volume centers were more likely to receive a lobectomy than those treated at intermediate- and lowvolume centers ( $17 \%$ vs $14 \%$ vs $12 \%$, respectively; $P=.01$ ). Among patients who received surgery, rates of R0 resections were higher at high-volume facilities $(21 \%)$ than at intermediate- (16\%) and low-volume (14\%) facilities. Patients treated at high-volume facilities were more likely to receive trimodality therapy than those treated at low- and intermediate-volume facilities ( $12 \%$ vs $9 \%$ each for lowand intermediate-volume facilities; $P<.001$ ) (Table 1).

In subgroup analysis, patients with $\mathrm{T} 4$ disease were more likely to receive surgical therapy at high-volume centers $(25 \%)$ than those treated at intermediate- $(22 \%)$ 
and low-volume centers (18\%). A similar trend was seen in patients with $\mathrm{N} 2$ disease: those treated at high-volume centers were more likely to receive surgery $(24 \%)$ than those treated at intermediate- (18\%) and low-volume (17\%) centers.

\section{Survival Analysis}

Cox multivariable analysis showed that female sex, black race, lower Charlson-Deyo comorbidity score, private insurance, lower tumor grade, and receipt of trimodality therapy (surgery + CRT) were associated with better survival (Table 2). In multivariate analysis, facility volume was independently associated with all-cause mortality. Compared with patients treated at high-volume facilities, those treated at intermediate- and lower-volume facilities had a significantly higher risk of death (hazard ratio [HR], 1.09; 95\% CI, 1.07-1.11; and HR, 1.11; 95\% CI, $1.09-1.13$, respectively).

Unadjusted median OS values by facility volume were 13, 15, and 16 months for low-, intermediate-, and high-volume centers, respectively $(P<.001)$. Patients treated at high-volume facilities had significantly better 1-, 3-, and 5-year OS than those treated at low-volume centers $(P<.001)$ (Figure 2, Table 4). Surgical therapy was independently associated with prolonged OS $(P<.001)$. Among patients who received surgery, those who underwent pneumonectomy had significantly higher risk of death than those who underwent lobectomy (HR, 1.37; 95\% CI, 1.30-1.45; $P<.0001$ ).

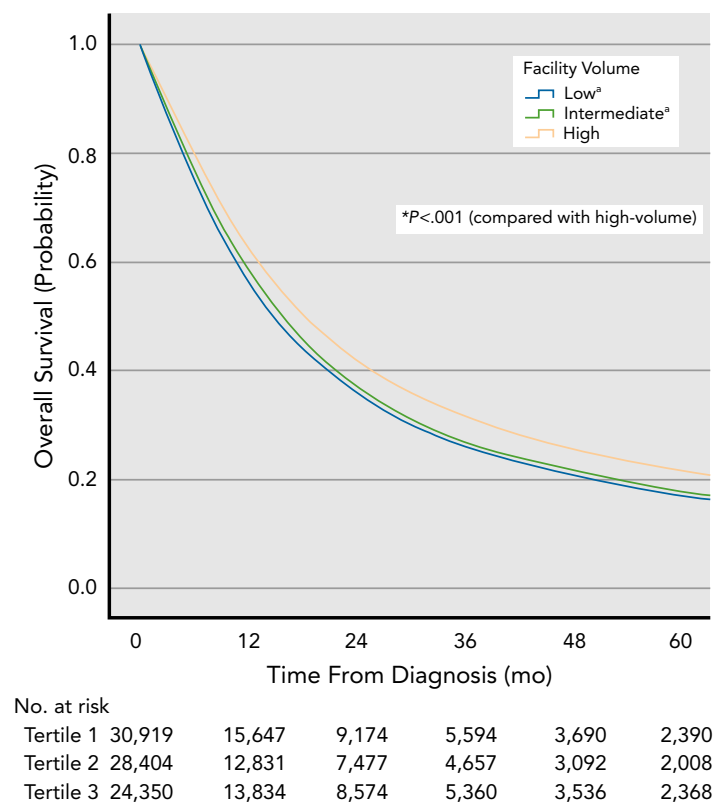

Figure 2. Kaplan-Meier analysis of overall survival based on hospital volume.

${ }^{a} P<.001$ (vs high-volume facility).
In subgroup analysis, patients with $\mathrm{T} 4$ disease managed with surgery at high-volume centers had better median OS (19 months) than those treated at intermediate- and low-volume centers (16 and 15 months, respectively; $P<.001$ ). A similar trend was seen in patients with $\mathrm{N} 2$ disease; patients treated at high-volume centers had a better median OS (18 months) than those treated at intermediate- and low-volume centers (16 and 14 months, respectively; $P<.001$ ). In addition, we compared outcomes related to different levels of facility volume by stratifying for upfront treatment approach, which showed that patients managed with surgery, chemotherapy only, or CRT at high-volume centers had a statistically significant median OS (see supplemental eTable 1, available with this article at JNCCN.org).

\section{Discussion}

This retrospective analysis using the NCDB, which includes $>70 \%$ of newly diagnosed cancer cases in the United States, showed that patients treated at facilities with higher annual volumes of stage IIIA NSCLC had lower mortality rates than those treated at lowervolume facilities. This difference persisted even after adjustment for patient demographics, functional status, tumor characteristics, treatment modality received, and clustering of the outcome within hospitals. Previous studies have demonstrated that patients who had surgery performed at high-volume centers and by dedicated thoracic surgeons had better survival than their counterparts. ${ }^{11,12}$

Our study evaluated the potential differences in treatment patterns among patients with stage IIIA NSCLC based on facility volume. We found that patients at high-volume centers were more likely to receive neoadjuvant and surgical therapies. This difference in treatment patterns may be attributed to better selection of patients for surgery at high-volume centers. Given the heterogeneity of stage IIIA disease, treatment algorithms and practices vary among hospitals. For example, some centers opt for surgical resection of the primary tumor if disease is limited to a single section of mediastinal nodes. ${ }^{11}$ Selection of patients for surgical resection in stage IIIA NSCLC is tricky because of the lack of precise definitions that can be applied universally; for example, most guidelines reserve surgery for earlier-T-stage (T1-T3), N0-N1 disease and "potentially" resectable stage IIIA-T4 or IIIA-N2 disease. The definition of "resectable" IIIA-T4 or IIIA-N2 disease is largely dependent on the local expertise of the care team. ${ }^{13}$

Moreover, the decision to proceed with surgical resection usually includes a detailed preoperative pulmonary function evaluation and critical intraoperative 
Table 4. Facility-Wise Treatment Modalities and 1-, 3-, and 5-Year Overall Survival

\begin{tabular}{|c|c|c|c|c|c|c|c|}
\hline Facility Volume & \multicolumn{4}{|c|}{ Therapy Received (\%) } & \multicolumn{3}{|c|}{ Survival (\%) } \\
\hline Low & 18 & 68 & 67 & 9 & 57 & 26 & 17 \\
\hline Intermediate & 20 & 69 & 68 & 9 & 59 & 27 & 18 \\
\hline High & 25 & 70 & 67 & 12 & 63 & 32 & 22 \\
\hline
\end{tabular}

assessment of the feasibility of complete resection, and considers the feasibility of handling the postoperative complications at the surgery-performing facility, which typically needs a multidisciplinary team approach. Our findings show that a larger percentage of patients with T4 and N2 disease were likely to receive surgery performed at high-volume centers. Furthermore, we found that patients with stage IIIA-T4 or IIIA-N2 disease managed surgically at high-volume centers had a better median OS. Although a proficient surgical team may be associated with better survival in stage IIIA NSCLC at high-volume facilities, there may be several other factors to consider in our analysis, including the availability of advanced diagnostic and staging techniques, a multidisciplinary management team that maintains effective communication, enrollment in clinical trials, ${ }^{14}$ and level of proficiency of the care team in managing any underlying pulmonary disease, which may not be readily available at low-volume facilities.

It is possible that a large number of patients with "lower disease burden" were treated in high-volume centers, but no clinically meaningful differences in tumor stage and nodal status were noted among high-, intermediate-, and low-volume centers (Table 1). Among patients who received surgery, those who underwent lobectomy had better survival than those who received pneumonectomy. Previous studies have shown similar superior survival rates in patients who received lobectomy, which may be attributed to higher complication rates associated with pneumonectomy or to pneumonectomy being performed in patients with extensive disease. ${ }^{15-17}$ Interestingly, patients managed with surgery at high-volume centers were more likely to receive lobectomy than those managed at low-volume centers ( $17 \%$ vs $12 \%$ ). A similar trend was seen in an Ontario registry-based study, which showed that high-volume surgeons were less likely to perform higher-risk pneumonectomy procedures compared with lower-volume surgeons. ${ }^{18}$

Proper selection of surgical procedure based on surgeon expertise may translate to a significant reduction in adverse events. Among patients who underwent surgical resection, those treated at higher-volume centers had better R0 resection rates. The association of better R0 resection rates with high-volume facilities in patients undergoing complex cancer surgery has been attributed to increased proficiency of the surgical teams at these facilities. The other possible explanation of better OS associated with high-volume facilities is the availability of a supportive care team providing better ancillary support and symptom management. ${ }^{19,20}$ These caveats illustrate the increasing complexity of stage IIIA NSCLC management and support our findings that the volume-outcome disparity is pronounced. In addition, Wang et $\mathrm{al}^{21}$ showed that patients with stage III NSCLC treated with definitive CRT had a better OS at highvolume centers.

Our study has considerable limitations that are inherent to retrospective registry-based studies. First, because the NCDB data are limited to the facility but not to individual practitioners, the study did not take annual physician volume/cumulative physician experience into consideration. However, facility volume, rather than individual practitioner volume, better reflects the multidisciplinary nature of contemporary stage IIIA NSCLC treatment. Second, owing to lack of detailed information about the hospitals and specific type of therapies (and their complications) offered to patients, we were not able to analyze specific reasons for high-volume facilities having better outcomes than low-volume facilities. Third, the NCDB reports OS but not cancerspecific survival, thus allowing the possibility that patients treated at high-volume facilities might be healthier at baseline. To address this concern, we adjusted the Charlson-Deyo comorbidity score in the analysis and did not see significant differences in this score among the hospital groups. Fourth, the NCDB does not provide data on the percentage of patients enrolled in the clinical trials at these facilities. It is possible that patients treated at high-volume facilities have an increased opportunity to participate in clinical trials, which can also affect outcomes. Finally, the NCDB does not capture any data if the patient switches institution after receiving the first therapy. Despite this, we believe our results provide a real-world view of the state of stage IIIA NSCLC management, and show that even after adjustment for treatment modality, patient volume was associated with outcomes. 
Results of our study raise several important considerations. It is important to note that not all patients with stage IIIA disease have access to high-volume facilities, for multiple reasons, which may include financial burden and physical effort needed to travel long distances to access high-volume hospitals. Our study emphasizes the need for future studies to determine what specific factors confer a survival advantage at highvolume facilities and to ascertain whether these factors can be adapted at low-volume facilities or whether the regionalization of care improves care delivery and survival in heterogeneous cancers such as stage IIIA NSCLC, which require multidisciplinary cancer treatment and complex procedures.

\section{Conclusions}

Our study adds to the mounting evidence of a volumeoutcome relationship in managing patients with cancer. After adjustment for patient demographics, tumor characteristics, therapy received, and comorbidity index, our results showed that patients treated for stage IIIA NSCLC at high-volume facilities had a significantly lower risk of all-cause mortality than those treated at lower-volume facilities. This finding is critical, especially for patients with stage IIIA NSCLC, because there is no consensus regarding optimal management, and a multidisciplinary approach is needed.

Submitted April 23, 2019; accepted for publication September 12, 2019

Previous presentation: This work was presented at the 2018 ASCO Annual Meeting; June 1-5, 2018; Chicago, Illinois.

Author contributions: Study concept: Kommalapati, Tella. Study design: Kommalapati, Tella, Appiah, Ganti. Data acquisition: Kommalapati, Tella. Data interpretation: Tella, Appiah, Smith. Manuscript preparation: Kommalapati, Tella, Ganti. Critical revision: All authors. Final manuscript approval: All authors.

Disclosures: The authors have not received any financial consideration from any person or organization to support the preparation, analysis, results, or discussion of this article.

Correspondence: Anuhya Kommalapati, MBBS, Palmetto Health USC Medical Group, University of South Carolina, 2 Medical Park, Suite 402, Columbia, SC 29203. Email: anuhya781@gmail.com

\section{References}

1. Siegel RL, Miller KD, Jemal A. Cancer statistics, 2018. CA Cancer J Clin 2018;68:7-30

2. American Cancer Society. Cancer Facts and Figures 2018. Available at: https://www.cancer.org/content/dam/cancer-org/research/cancer-factsand-statistics/annual-cancer-facts-and-figures/2018/cancer-facts-andfigures-2018.pdf. Accessed March 1, 2018.

3. Biswas T, Sharma N, Machtay M. Controversies in the management of stage III non-small-cell lung cancer. Expert Rev Anticancer Ther 2014 14:333-347.

4. Patel AP, Crabtree TD, Bell JM, et al. National patterns of care and outcomes after combined modality therapy for stage IIIA non-small-cell lung cancer. J Thorac Oncol 2014;9:612-621.

5. Berry MF, Worni M, Pietrobon R, et al. Variability in the treatment of elderly patients with stage IIIA (N2) non-small cell lung cancer. J Thorac Oncol 2013;8:744-752.

6. Albain KS, Swann RS, Rusch VW, et al. Radiotherapy plus chemotherapy with or without surgical resection for stage III non-small-cell lung cancer: a phase III randomised controlled trial. Lancet 2009;374:379-386.

7. Van Schil PE, Berzenji L, Yogeswaran SK, et al. Surgical management of stage IIIA non-small cell lung cancer. Front Oncol 2017;7:249.

8. Kumar SS, Higgins KA, McGarry RC. Emerging therapies for stage III nonsmall cell lung cancer: stereotactic body radiation therapy and immunotherapy. Front Oncol 2017;7:197

9. Birkmeyer JD, Siewers AE, Finlayson EV, et al. Hospital volume and surgical mortality in the United States. N Engl J Med 2002;346: 1128-1137.

10. Santana-Davila R, Martins R. Treatment of stage IIIA non-small-cell lung cancer: a concise review for the practicing oncologist. J Oncol Pract 2016; 12:601-606.

11. Bach PB, Cramer LD, Schrag D, et al. The influence of hospital volume on survival after resection for lung cancer. N Engl J Med 2001;345:181-188.

12. Farjah F, Flum DR, Varghese TK Jr, et al. Surgeon specialty and long-term survival after pulmonary resection for lung cancer. Ann Thorac Surg 2009; 87:995-1004, discussion 1005-1006.

13. Van Schil PE. Stage IIIA-N2 non-small-cell lung cancer: from 'surprise' involvement to surgical nightmare. Eur J Cardiothorac Surg 2016;49: 1613-1614

14. Eaton BR, Pugh SL, Bradley JD, et al. Institutional enrollment and survival among NSCLC patients receiving chemoradiation: NRG Oncology Radiation Therapy Oncology Group (RTOG) 0617. J Natl Cancer Inst 2016 108:djw034.

15. Myrdal G, Gustafsson G, Lambe M, et al. Outcome after lung cancer surgery. Factors predicting early mortality and major morbidity. Eur J Cardiothorac Surg 2001;20:694-699.

16. Shi W, Zhang W, Sun H, et al. Sleeve lobectomy versus pneumonectomy for non-small cell lung cancer: a meta-analysis. World J Surg Oncol 2012;10:265.

17. Lee ES, Park SI, Kim YH, et al. Comparison of operative mortality and complications between bronchoplastic lobectomy and pneumonectomy in lung cancer patients. J Korean Med Sci 2007;22:43-47.

18. Camposilvan I, Akhtar-Danesh N, Schneider L, et al. The effect of surgeon volume on procedure selection in non-small cell lung cancer surgeries. J Thorac Cardiovasc Surg 2015;150:507-513.

19. Finks JF, Osborne NH, Birkmeyer JD. Trends in hospital volume and operative mortality for high-risk surgery. N Engl J Med 2011;364: 2128-2137.

20. Al-Sahaf $\mathrm{M}$, Lim $\mathrm{E}$. The association between surgical volume, survival and quality of care. J Thorac Dis 2015;7(Suppl 2):S152-155.

21. Wang $E H$, Rutter $C E$, Corso $C D$, et al. Patients selected for definitive concurrent chemoradiation at high-volume facilities achieve improved survival in stage III non-small-cell lung cancer. J Thorac Oncol 2015;10: 937-943. 